\title{
CONSTANT-LENGTH SUBSTITUTIONS AND COUNTABLE SCRAMBLED SETS
}

\author{
FRANÇOIS BLANCHARD, FABIEN DURAND, AND ALEJANDRO MAASS
}

\begin{abstract}
In this paper we provide examples of topological dynamical systems having either finite or countable scrambled sets. In particular we study conditions for the existence of Li-Yorke, asymptotic and distal pairs in constant-length substitution dynamical systems. Starting from a circle rotation we also construct a dynamical system having Li-Yorke pairs, none of which is recurrent.
\end{abstract}

\section{INTRODUCTION}

One definition of topological chaos, based on ideas in $[\mathrm{LY}$, emerged twenty-five years ago. It is not the only definition of chaos by far. It relies on the existence of uncountable 'scrambled' subsets. Here we investigate what might be called 'the edge of Li-Yorke chaos': we give examples of systems having only finite or countable scrambled subsets. In particular those are examples of transitive dynamical systems of zero topological entropy which are not Li-Yorke chaotic.

Let $(X, T)$ be a topological dynamical system: $X$ is a compact metric space with metric $\varrho$, and $T$ is a surjective continuous map from $X$ to itself. A pair of points $\{x, y\} \subseteq X$ is said to be a $L i-$ Yorke pair if one has simultaneously

$$
\limsup _{n \rightarrow \infty} \varrho\left(T^{n} x, T^{n} y\right)>0 \text { and } \liminf _{n \rightarrow \infty} \varrho\left(T^{n} x, T^{n} y\right)=0 .
$$

A set $S \subseteq X$ is called scrambled if any pair of distinct points $\{x, y\} \subseteq S$ is a LiYorke pair. Finally, a system $(X, T)$ is called chaotic in the sense of Li and Yorke if $X$ contains an uncountable scrambled set. Li-Yorke chaos has been recently proved to result from various dynamical properties: positive entropy; 2 -scattering; transitivity together with one periodic orbit (see [BGKM] and [HY]). The opposite situation exists too: equicontinuous and distal systems have no Li-Yorke pairs. The aim of this article is to describe various systems that are not $\mathrm{Li}-$ Yorke chaotic while having Li-Yorke pairs. Most of them arise from constant-length substitutions, which are the most classical topological extensions of odometers.

A pair $\{x, y\} \subseteq X$ is called distal if $\liminf _{n \rightarrow \infty} \varrho\left(T^{n} x, T^{n} y\right)>0$. If, instead, $\lim \inf _{n \rightarrow \infty} \varrho\left(T^{n} x, T^{n} y\right)=0$ then the pair is called proximal. If the limit exists and is equal to zero then the pair is called asymptotic. Thus $\{x, y\}$ is a Li-Yorke pair if and only if it is proximal but not asymptotic. The sets of distal, proximal and asymptotic pairs of $(X, T)$ are denoted by $\mathbf{D}(X, T), \mathbf{P}(X, T)$ and $\mathbf{A}(X, T)$ respectively. Clearly the set of $\mathrm{Li}$-Yorke pairs is $\mathbf{L Y}(X, T)=\mathbf{P}(X, T) \backslash \mathbf{A}(X, T)$. The sets of distal pairs, Li-Yorke pairs and asymptotic pairs partition $X^{2}$. It is easy to see that the image of a proximal (asymptotic) pair under a factor map is

1991 Mathematics Subject Classification. Primary: 58F08; Secondary: 58F03,54H20.

Key words and phrases. substitution, Li-Yorke pairs, topological dynamics. 
proximal (asymptotic). Observe that in all these definitions the order of a pair is irrelevant: a non-diagonal pair may be considered as a subset of cardinality 2 . Finally we point out that even if definitions of distal, proximal, asymptotic and Li-Yorke pairs work for any general dynamical system $(X, T)$, in the examples that will follow we essentially look to the case where $T$ is a homeomorphism.

A dynamical system $(X, T)$ is called minimal if the unique closed invariant subsets of $X$ are $X$ and $\emptyset$. A distal dynamical system is one in which every non-diagonal pair is distal. A dynamical system having no Li-Yorke pairs is called almost distal BGKM]; Sturmian systems and the Morse system are elementary examples. In AA Akin and Auslander introduce strong Li-Yorke pairs, i.e., those Li-Yorke pairs that are recurrent under $T \times T$. They call semi-distal a system without strong Li-Yorke pairs. Distal, almost distal and semi-distal systems are minimal when transitive. A system described some time ago by Floyd ([Au , p. 26) was recently remarked to be semi-distal but not almost distal ([Y], AA]); it is an extension of an adding machine in which fibers are intervals or singletons. Here we give several examples with the same property, one comes from a substitution of constant length and the other is a bounded-to-one extension of an irrational rotation.

In Section 3 we study distal, asymptotic and Li-Yorke pairs in systems generated by constant-length substitutions; this study turns out to be complete when the alphabet has size 2. This yields examples of systems in which scrambled sets have any given finite cardinality. The following section is devoted to the construction of an inverse limit of substitution systems having the property that all scrambled sets are at most countable. Finally in Section 5 we construct a new semi-distal, not almost distal, system.

\section{Preliminaries about substitutions}

2.1. General facts about substitutions. A substitution is a map $\tau: A \rightarrow A^{+}$, where $A$ is a finite set and $A^{+}$is the set of finite sequences with values in $A ; \tau$ is a substitution of constant length $p, p \geq 2$, if $|\tau(a)|=p$ for any $a \in A$, where $|\cdot|$ denotes the length of a word.

In the sequel $\mathbb{N}$ stands for the set of non-negative integers. The sets of one-sided and two-sided infinite sequences are denoted by $A^{\mathbb{N}}$ and $A^{\mathbb{Z}}$ respectively. If $K=\mathbb{N}$ or $\mathbb{Z}$, points of $A^{K}$ are denoted by $\left(x_{i}\right)_{i \in K}$ and given $i, j \in K, i \leq j, x(i, j)$ denotes the word (or sub-word) $x_{i} \ldots x_{j}$ of $x$; by convention $x(i, i)=x_{i}$. For a word $w \in A^{+}$ if $|w|=n$ we put $w=w_{0} \ldots w_{n-1}$ and $w(i, j)=w_{i} \ldots w_{j}$ for $0 \leq i \leq j<n$. The shift map $T: A^{K} \rightarrow A^{K}$ is the continuous map defined by $T\left(\left(x_{i}\right)_{i \in K}\right)=\left(x_{i+1}\right)_{i \in K}$. A subshift $X$ is a closed $T$-invariant subset of $A^{\mathbb{N}}$ or $A^{\mathbb{Z}}$; the action we consider on $X$ will always be the restriction of the shift map to $X$ and will also be denoted by $T$. In this paper we will look exclusively at the case where $K=\mathbb{Z}$; in this case $T$ is a homeomorphism. We say that a word appears in a subshift or is a sub-word of a subshift if it is a sub-word of a point of the subshift. A subshift is completely determined by the list of all words that never occur as sub-words of its points.

The substitution $\tau$ can be naturally extended by concatenation to $A^{+}, A^{\mathbb{N}}$ and $A^{\mathbb{Z}}$; for $x=\left(x_{i}\right)_{i \in \mathbb{Z}} \in A^{\mathbb{Z}}$ the extension is given by

$$
\tau(x)=\ldots \tau\left(x_{-2}\right) \tau\left(x_{-1}\right) \cdot \tau\left(x_{0}\right) \tau\left(x_{1}\right) \ldots
$$


where the central dot separates negative and non-negative coordinates of $\tau(x)$. A further natural convention is that the image of the empty word $\varepsilon$ is $\varepsilon$. We say that $\tau$ is primitive if there is $n \in \mathbb{N}$ such that $a$ appears in $\tau^{n}(b)$ for every $a, b \in A$. The substitution $\tau$ generates a subshift $X_{\tau}$ of $A^{\mathbb{Z}}$ which is the smallest subshift of $A^{\mathbb{Z}}$ admitting all words $\left\{\tau^{n}(a): n \in \mathbb{N}, a \in A\right\}$; when $\tau$ is primitive the subshift it generates is minimal. For more details and complements about this subsection we refer the reader to $\mathrm{Q}$. To end this subsection we recall the following result due to B. Mossé [Mo] (see also [MS]).

Theorem 2.1. Let $\tau$ be a primitive substitution. Suppose $X_{\tau}$ is infinite. Then, $\tau: X_{\tau} \rightarrow \tau\left(X_{\tau}\right)$ is a one-to-one continuous map. If $\tau$ is of constant length $p$, then $T^{p} \circ \tau=\tau \circ T$ and $\tau\left(X_{\tau}\right)$ is a proper p-periodic subset of $X_{\tau}$, that is, $\left\{T^{i}\left(\tau\left(X_{\tau}\right)\right)\right.$ : $i \in\{0, \ldots, p-1\}\}$ is a clopen (closed and open) partition of $X_{\tau}$.

In the case where $\tau$ is of constant length $p$ the partition $\left\{T^{i}\left(\tau\left(X_{\tau}\right)\right): i \in\{0, \ldots, p-\right.$ $1\}$ \} will be called the fundamental partition of $X_{\tau}$.

Let $\tau$ be a primitive substitution. Let us now recall a way to prove that $X_{\tau}$ is infinite or not. J.-J. Pansiot $\mathrm{Pa}$, and, T. Harju and M. Linna HL proved (in our settings) that it is decidable whether $X_{\tau}$ is infinite or not. We need some definitions. Let $L(\tau)$ be the set of finite words having an occurrence in some $x \in X_{\tau}$. We say a word $u \in L\left(X_{\tau}\right)$ is biprolongeable (with respect to $\left.X_{\tau}\right)$ if there exist two distinct letters $a$ and $b$ such that $u a$ and $u b$ belong to $L\left(X_{\tau}\right)$. We say $\tau: A \rightarrow A^{+}$is simplifiable if there exist an alphabet $B,|B|<|A|$, and two morphisms $f: A^{*} \rightarrow B^{*}, g: B^{*} \rightarrow A^{*}$ such that $\tau=g \circ f$. The substitution $\tau$ is elementary if it is not simplifiable. Note that when $|A|=2, \tau$ is simplifiable if and only if there exist $u \in A^{+}, n, m \in \mathbb{N}$ such that $\tau(A)=\left\{u^{n}, u^{m}\right\}$.

Proposition 2.2. $\mathrm{Pa}$ Let $\tau: A \rightarrow A^{+}$be an elementary primitive substitution. Then, $X_{\tau}$ is infinite if and only if there exists at least one biprolongeable letter $a \in A$.

If $\tau$ is not elementary then there exist an alphabet $B,|B|<|A|$, and two morphisms $f: A^{*} \rightarrow B^{*}, g: B^{*} \rightarrow A^{*}$ such that $\tau=g \circ f$. Let $\gamma=f \circ g$. We can remark that $X_{\gamma}$ is infinite if and only if $X_{\tau}$ is infinite.

If $\gamma$ is elementary we apply Proposition 2.2 to know if $X_{\tau}$ is infinite. Otherwise we continue the induction. It will stop because the sequence of the cardinality of the alphabets we produce is decreasing and because when $|A|=1, X_{\tau}$ is finite.

2.2. One-to-one reduction of substitutions. It is often very convenient to assume that a substitution is one-to-one. Here we show that this can be done without any serious loss of generality.

Let $\tau: A \rightarrow A^{+}$be a substitution, and let $B \subset A$ be such that for any $a \in A$ there is a unique $b \in B$ for which $\tau(a)=\tau(b)$. We define the onto map $\phi: A \rightarrow B$ by $\phi(a)=b$ if $\tau(a)=\tau(b)$. We call $\bar{\tau}: B \rightarrow B^{+}$the unique substitution satisfying $\bar{\tau} \circ \phi=\phi \circ \tau$. If $\tau$ is primitive then $\bar{\tau}$ is primitive too. We say $\bar{\tau}$ is a reduction of $\tau$. The map $\phi$ defines a topological factor from $\left(X_{\tau}, T\right)$ onto $\left(X_{\bar{\tau}}, T\right)$, also called $\phi$. One checks that $\tau(\phi(a))=\tau(a)$ for all $a \in A$; thus $\tau(\phi(x))=\tau(x)$ for all $x \in X_{\tau}$.

Proposition 2.3. Let $\tau: A \rightarrow A^{+}$be a primitive substitution. The set $X_{\tau}$ is finite if and only if $X_{\bar{\tau}}$ is finite. Moreover, if $X_{\tau}$ is not finite then $X_{\tau}$ is topologically conjugate to $X_{\bar{\tau}}$. 
Proof. If $X_{\tau}$ is finite $X_{\bar{\tau}}$ is finite too. On the other hand if $X_{\bar{\tau}}$ is finite all its points are periodic for the shift. Therefore $\phi(x)$ is periodic and $\tau(x)=\tau(\phi(x))$ too for $x \in X_{\tau}$. Since $X_{\tau}$ is minimal and $\tau(x) \in X_{\tau}$ one concludes that $X_{\tau}$ is finite. Suppose $X_{\tau}$ is not finite. Since $\phi$ is a factor map we only need to prove $\phi$ is one-to-one. If $\phi(x)=\phi(y), x, y \in X_{\tau}$, then $\tau(x)=\tau(\phi(x))=\tau(\phi(y))=\tau(y)$. But in this case $\tau: X_{\tau} \rightarrow \tau\left(X_{\tau}\right)$ is one-to-one (Theorem 2.1) and $x=y$. Therefore $\phi$ is a topological conjugacy.

Remark that if $X_{\tau}$ is finite then $\left(X_{\tau}, T\right)$ is not conjugate to $\left(X_{\bar{\tau}}, T\right)$. For example take $\tau:\{0,1\} \rightarrow\{0,1\}^{+}, \tau(0)=\tau(1)=01$. A substitution $\tau: A \rightarrow A^{+}$is said to be one-to-one if for all $a, b \in A, a \neq b, \tau(a) \neq \tau(b) ; \tau$ is one-to-one if and only if $|B|=|A|$. In this case $\tau$ and $\bar{\tau}$ are the same. If $\tau$ is not one-to-one then $|B|<|A|$. By repeating the reduction described above finitely many times we obtain a oneto-one substitution $\sigma: C \rightarrow C^{+}$and a factor map $\psi:\left(X_{\tau}, T\right) \rightarrow\left(X_{\sigma}, T\right)$, which is a conjugacy when $X_{\tau}$ is not finite. The substitution $\sigma$ is called the one-toone reduction of $\tau$ (since it is uniquely defined up to the rename of letters in the alphabet).

Note that when $X_{\tau}$ is finite, the one-to-one reduction can be defined on an alphabet which is not a singleton. It is the case for $\tau:\{0,1\} \rightarrow\{0,1\}^{+}$defined by $\tau(0)=010$ and $\tau(1)=101$ where $X_{\tau}$ is an orbit of period two.

2.3. Constant-length substitutions and odometers. Let $p \geq 2$ be an integer. The inverse limit $\mathcal{O}_{p}$ of the sequence of groups $\left(\mathbb{Z} / p^{k} \mathbb{Z}: k \in \mathbb{N}\right)$ endowed with the addition of 1 is called the $p$-odometer; $\mathcal{O}_{P}$ is a compact topological ring. Odometers are always minimal and uniquely ergodic. The $p$-odometer will be denoted by $\left(\mathcal{O}_{p}, T_{0}\right)$. With $[p]=\{0, \ldots, p-1\}$ there is a natural homeomorphism $n_{p}:[p]^{\mathbb{N}} \rightarrow \mathcal{O}_{p}$ such that $n_{p}(\delta)=\sum_{i \geq 0} \delta_{i} p^{i}$ for $\delta=\left(\delta_{i}\right)_{i \in \mathbb{N}} \in[p]^{\mathbb{N}}$. The sequence $\delta$ is the expansion in base $p$ of number $n_{p}(\delta)$. Through this homeomorphism we will identify $[p]^{\mathbb{N}}$ with $\mathcal{O}_{p}$. The integers can be identified with the integer multiples of the identity, and so, we can think in $\mathbb{Z}$ as a dense subring of $\mathcal{O}_{p}$. A number in $\mathcal{O}_{p}$ is positive (respectively negative) integer if and only if its expansion sequence is eventually 0 (resp. eventually $p-1$ ). For addition in $\mathcal{O}_{p}$ you add the digits modulo $p$ but with the carry to the right. The projection to $\mathbb{Z}_{p^{k}}$ is given by the list of the first $k$ digits. Let $\tau: A \rightarrow A^{+}$be a primitive substitution of constant length $p$ such that $X_{\tau}$ is not finite. The $p$-odometer is a factor of the subshift $X_{\tau}$. The proof of this fact is based on the following central result of the theory of substitutions.

Theorem 2.4 ([De,, $\mathrm{Mo}])$. Let $\tau$ be a primitive substitution such that $X_{\tau}$ is not finite. Then for any $x \in X_{\tau}$ there are a unique sequence of points $\left(x^{(i)}\right)_{i \in \mathbb{N}} \subseteq X_{\tau}$ and a unique sequence of positive integers $\left(\delta_{i}\right)_{i \in \mathbb{N}} \subseteq[p]$ such that $x^{(0)}=x$ and $\tau\left(x^{(i)}\right)=T^{-\delta_{i-1}}\left(x^{(i-1)}\right), i \geq 1$. Moreover, the map $\pi: X_{\tau} \rightarrow[p]^{\mathbb{N}}$ defined by $\pi(x)=\left(\delta_{i}\right)_{i \in \mathbb{N}}$ is continuous.

Proof. We sketch part of the proof. The fundamental partition is naturally indexed by $[p]$, that is, to $j \in[p]$ we associate $T^{j}\left(\tau\left(X_{\tau}\right)\right)$. The point $x=x^{(0)}$ lies in a unique member of the partition with index $\delta_{0}$. Pull back by $T^{\delta_{0}}$ to get an element of $\tau\left(X_{\tau}\right)$. Then apply $\tau^{-1}$ to obtain the element $x^{(1)}$. Continue inductively.

For $i \in \mathbb{N}$ and $x \in X_{\tau}$ put $p_{i}(x)=x^{(i)}\left(-\delta_{i},-1\right)$ and $s_{i}(x)=x^{(i)}\left(1, p-1-\delta_{i}\right)$. If $\delta_{i}=p-1$ we put $s_{i}(x)$ to be the empty word. Analogously if $\delta_{i}=0$ let $p_{i}(x)$ be 
the empty word. From Theorem 2.4 for all $i \geq 0$ one has

$$
\tau\left(x_{0}^{(i+1)}\right)=p_{i}(x) x_{0}^{(i)} s_{i}(x)
$$

and

$$
\begin{array}{ll}
\left|p_{i}(T x)\right|=0 & \text { if } i<i^{*}, \\
\left|p_{i}(T x)\right|=\left|p_{i}(x)\right|+1 & \text { if } i=i^{*}, \\
\left|p_{i}(T x)\right|=\left|p_{i}(x)\right| & \text { if } i>i^{*},
\end{array}
$$

where $i^{*}=\inf \left\{i \in \mathbb{N}: \delta_{i} \neq p-1\right\}$. Set $\mathcal{I}^{-}(x)=\left\{i \in \mathbb{N}:\left|p_{i}(x)\right| \neq 0\right\}$ and $\mathcal{I}^{+}(x)=\left\{i \in \mathbb{N}:\left|s_{i}(x)\right| \neq 0\right\}$. By definition if $\pi(x)=\pi(y)$ then $\mathcal{I}^{-}(x)=\mathcal{I}^{-}(y)$ and $\mathcal{I}^{+}(x)=\mathcal{I}^{+}(y)$.

We will frequently look at pairs $(x, y)$ such that $\pi(x)=\pi(y)$. Two such points have a common expansion in base $p$ sequence $\left(\delta_{i}\right)_{i \in \mathbb{N}}$. In particular it follows that $\left|p_{i}(x)\right|=\left|p_{i}(y)\right|,\left|s_{i}(x)\right|=\left|s_{i}(y)\right|, i \in \mathbb{N}$, and $\mathcal{I}^{ \pm}(x)=\mathcal{I}^{ \pm}(y)$. In this case we will let $\mathcal{I}=\mathcal{I}^{+}(x)=\mathcal{I}^{+}(y)$ or $\mathcal{I}=\mathcal{I}^{-}(x)=\mathcal{I}^{-}(y)$. While the definitions are symmetric, we are interested in long-term positive time behavior and so our attention will focus on $\mathcal{I}^{+}$and $s_{i}$.

Lemma 2.5. Let $x \in X_{\tau}$. Then

$$
\tau^{i}\left(p_{i}(x)\right) \ldots \tau^{2}\left(p_{2}(x)\right) \tau\left(p_{1}(x)\right) p_{0}(x) . x_{0} s_{0}(x) \tau\left(s_{1}(x)\right) \tau^{2}\left(s_{2}(x)\right) \ldots \tau^{i}\left(s_{i}(x)\right)
$$

is the sub-word of $x$ centered in $x_{0}$ and $T^{-\left(\delta_{0}+\delta_{1} p+\cdots+\delta_{i} p^{i}\right)} x=\tau^{i+1}\left(x^{(i+1)}\right)$ for all $i \geq 0$.

Proof. This follows inductively from the definition of $\left(x^{(i)}\right)_{i \in \mathbb{N}}$ and $\left(\delta_{i}\right)_{i \in \mathbb{N}}$.

Define $k^{-}(x)=\max \left\{\left|\tau^{i}\left(p_{i}(x)\right) \ldots \tau^{2}\left(p_{2}(x)\right) \tau\left(p_{1}(x)\right) p_{0}(x)\right|: i \in \mathcal{I}^{-}(x)\right\}$ and $k^{+}(x)=$ $\max \left\{\left|x_{0} s_{0}(x) \tau\left(s_{1}(x)\right) \tau^{2}\left(s_{2}(x)\right) \ldots \tau^{i}\left(s_{i}(x)\right)\right|: i \in \mathcal{I}^{+}(x)\right\}$. Observe that $k^{-}(\cdot)$ and $k^{+}(\cdot)$ are constants on fibers of $\pi$. Also $k^{+}(x)$ is finite when $\mathcal{I}^{+}(x)$ is a finite set which is when the expansion in base $p$ of $\pi(x)$ is a negative integer in $\mathcal{O}_{p}$. Furthermore, if $\left|\mathcal{I}^{+}(x)\right|<\infty$ there is $j_{0} \in \mathbb{N}$ such that $\delta_{j}=p-1$ for $j \geq j_{0}$. Therefore $\left|s_{j}\left(T^{k^{+}(x)}(x)\right)\right|=p-1$ for $j \geq 0$ and

$$
\begin{aligned}
x(0, \infty) & =x(0, k-1) x(k) s_{0}\left(T^{k}(x)\right) \tau\left(s_{1}\left(T^{k}(x)\right)\right) \tau^{2}\left(s_{2}\left(T^{k}(x)\right)\right) \ldots \\
& =x(0, k-1) \lim _{m \rightarrow \infty} \tau^{m}\left(x_{1}^{(m)}\right),
\end{aligned}
$$

where $k=k^{+}(x)$. Analogously if $\left|\mathcal{I}^{-}(x)\right|<\infty$ for all $j \geq j_{0}, \delta_{j}=0$ and

$$
\begin{aligned}
x(-\infty, 0) & =\ldots \tau\left(p_{1}\left(T^{-k-1}(x)\right)\right) p_{0}\left(T^{-k-1}(x)\right) x(-k-1) x(-k, 0) \\
& =\lim _{m \rightarrow \infty} \tau^{m}\left(x_{-1}^{(m)}\right) x(-k, 0),
\end{aligned}
$$

where $k=k^{-}(x)$. Clearly $\left|\mathcal{I}^{+}(x)\right|$ and $\left|\mathcal{I}^{-}(x)\right|$ cannot be finite at the same time.

Let $u$ and $v$ be two words of $A^{+}$. Set

$$
[u . v]=\left\{\left(x_{n}\right)_{n \in \mathbb{Z}} \in X_{\tau} ; x_{-|u|} x_{-|u|+1} \ldots x_{-1}=u, x_{0} x_{1} \ldots x_{|v|-1}=v\right\} .
$$

This is a particular cylinder set where $u$ occurs just before the zero coordinate and $v$ begins immediately afterwards.

Lemma 2.6. Let $\left(a_{n} b_{n} c_{n}\right)_{n \in \mathbb{N}}$ be a sequence on $A^{3}$ and $\left(d_{n}\right)_{n \in \mathbb{N}}$ be a sequence of integers such that $0 \leq d_{n} \leq p^{n}-1$. Then

$$
\# \bigcap_{n \in \mathbb{N}} T^{d_{n}} \tau^{n}\left(\left[a_{n} \cdot b_{n} c_{n}\right]\right) \leq 1 .
$$


The proof is left to the reader.

Lemma 2.7. The map $\pi:\left(X_{\tau}, T\right) \rightarrow\left(\mathcal{O}_{p}, T_{0}\right)$ is a factor map such that for every $\delta \in \mathcal{O}_{p},\left|\pi^{-1}(\{\delta\})\right|<K$, where $K=\mid\left\{\right.$ words of length 3 of $\left.X_{\tau}\right\} \mid$.

Proof. It is clear by (2.2) that $T_{0} \circ \pi=\pi \circ T$, and $\pi$ is continuous by Theorem 2.4. Therefore, since $\left(X_{\tau}, T\right)$ is minimal, $\left(\pi\left(X_{\tau}\right), T_{0}\right)$ is a minimal system and $\pi\left(X_{\tau}\right)=\mathcal{O}_{p}$. This proves that $\pi$ defines a factor map. Let $\delta=\left(\delta_{i}\right)_{i \in \mathbb{N}} \in$ $\mathcal{O}_{p}$ and suppose $\left|\pi^{-1}(\{\delta\})\right| \geq K+1$. By Theorem 2.4 and Lemma 2.5. for all $x \in \pi^{-1}(\{\delta\})$ there exists a unique sequence of points $\left(x^{(n)}\right)_{n \geq 1}$ such that $x=T^{\left(\delta_{0}+p \delta_{1}+\cdots+p^{n} \delta_{n}\right)} \tau^{n+1}\left(x^{(n+1)}\right)$ for all $n \in \mathbb{N}$. Let $E=\left\{x_{1}, x_{2}, \ldots, x_{K+1}\right\} \subset$ $\pi^{-1}(\{\delta\})$. Since $|E|>K$, for all $n \geq 1$ there exists $z_{1} \neq z_{2} \in E$ such that $z_{1}^{(n)}(-1,1)=z_{2}^{(n)}(-1,1) \in A^{3}$. Then as $E$ is finite there are $x \neq y \in E$ and a strictly increasing sequence of integers $\left(n_{i}\right)_{i \in \mathbb{N}}$ such that $x^{\left(n_{i}\right)}(-1,1)=y^{\left(n_{i}\right)}(-1,1)$ for all $i \in \mathbb{N}$. Consequently $x$ and $y$ belong to

$$
T^{\left(\delta_{0}+p \delta_{1}+\cdots+p^{n_{i}-1} \delta_{n_{i}-1}\right)} \tau^{n_{i}}\left[x_{-1}^{\left(n_{i}\right)} \cdot x_{0}^{\left(n_{i}\right)} x_{1}^{\left(n_{i}\right)}\right] .
$$

Lemma 2.6 implies that $x=y$, which contradicts the choice of $x$ and $y$. Thus $\left|\pi^{-1}(\{\delta\})\right| \leq K$.

\section{Li-Yorke PAIRS OF CONSTANT-LENGTH SUBSTITUTION SYSTEMS}

Let $\tau: A \rightarrow A^{+}$be a primitive substitution of constant length $p \geq 2$ such that $X_{\tau}$ is not finite. The odometer is a distal system (actually an isometry); whenever $x, y \in X_{\tau}$ and $\pi(x) \neq \pi(y)$ one has $(x, y) \in \mathbf{D}\left(X_{\tau}, T\right)$, where $\pi$ is the factor map defined in Theorem 2.4. This implies that all Li-Yorke pairs of $\left(X_{\tau}, T\right)$, and consequently all scrambled sets, are contained in fibres $\pi^{-1}(\{\delta\}), \delta \in \mathcal{O}_{p}$. Then by Lemma 2.7 all scrambled sets have finite cardinality. For the same reason an asymptotic pair is included in one fiber.

A direct consequence of Proposition 2.3 is the following corollary.

Corollary 3.1. Let $\tau: A \rightarrow A^{+}$be a primitive substitution of constant length $p \geq 2$; let $\sigma$ be its one-to-one reduction and $\psi$ be the corresponding map. If $X_{\tau}$ is not finite

(1) $\psi \times \psi\left(\mathbf{A}\left(X_{\tau}, T\right)\right)=\mathbf{A}\left(X_{\sigma}, T\right)$,

(2) $\psi \times \psi\left(\mathbf{L Y}\left(X_{\tau}, T\right)\right)=\mathbf{L Y}\left(X_{\sigma}, T\right)$,

(3) $\psi \times \psi\left(\mathbf{D}\left(X_{\tau}, T\right)\right)=\mathbf{D}\left(X_{\sigma}, T\right)$.

(4) $\psi \times \psi\left(\mathbf{P}\left(X_{\tau}, T\right)\right)=\mathbf{P}\left(X_{\sigma}, T\right)$.

Also, $\psi$ maps $\tau$ to $\sigma$.

Therefore to study asymptotic, proximal, distal and Li-Yorke pairs of $\left(X_{\tau}, T\right)$ it is enough to consider the one-to-one reduced system $\left(X_{\sigma}, T\right)$.

Definition 3.2. (1) Let $u, u^{\prime} \in A^{+}$be two words of the same length. We say they have a coincidence if $u_{i}=u_{i}^{\prime}$ for some coordinate $i \in\{0, \ldots,|u|-1\}$. All $i \in\{0, \ldots,|u|-1\}$ such that $u_{i} \neq u_{i}^{\prime}$ are called non-coincidences.

(2) Let $\tau: A \rightarrow A^{+}$be a substitution of constant length $p$. We say that $\tau$ has $a$ coincidence if there are $a, b \in A, a \neq b$, such that $\tau(a)$ and $\tau(b)$ have $a$ coincidence. We say it has overall coincidences if the last property holds for any $a, b \in A$. We say it has partial coincidence if there exist coincidences but no overall coincidences. 
When $A=\{0,1\}$ and $\tau$ has coincidences, then it has overall coincidences.

Proposition 3.3. Let $\tau$ be a primitive substitution and assume $X_{\tau}$ is infinite. If $\tau$ has overall coincidences then each pair $\{x, y\}, x \neq y$, with $\pi(x)=\pi(y)$ is proximal.

Proof. Let $\{x, y\}, x \neq y$, be a pair such that $\pi(x)=\pi(y)$. Shift so as to have $\mathcal{I}$ infinite. Applying Lemma 2.5 we see the lengths of the $s_{i}$ agree. A coincidence occurs within $\tau\left(s_{i}(x)\right)$ and $\tau\left(s_{i}(y)\right)$ whenever $\delta_{i} \neq p-1$. Thus, there is a common block of length $p^{i-1}$ between $\tau^{i}\left(s_{i}(x)\right)$ and $\tau^{i}\left(s_{i}(y)\right)$.

In the case of the previous proposition, the Li-Yorke pairs are exactly those pairs in a fiber which are not asymptotic.

It is well known that any infinite subshift has non-trivial asymptotic pairs. Moreover, it has been proved in $\mathrm{Q}$ that the number of different orbits of asymptotic pairs in the Cartesian product is finite (see also [HZ]). This property is also true for any infinite subshift with sub-affine symbolic complexity. In [BDH the authors give an upper bound for the number of different orbits of asymptotic pairs for substitution subshifts. In the next propositions we show how asymptotic and Li-Yorke pairs arise in our substitution systems.

Proposition 3.4. Let $\tau: A \rightarrow A^{+}$be a one-to-one primitive substitution of constant length $p$ such that $X_{\tau}$ is not finite. Let $\{x, y\}$ be a pair such that $\pi(x)=$ $\pi(y)$ and let $\mathcal{I}=\mathcal{I}^{+}(x)=\mathcal{I}^{+}(y)$ and $k=k^{+}(x)=k^{+}(y)$. Then

(1) $(x, y) \in \mathbf{A}\left(X_{\tau}, T\right)$ if and only if either $\mathcal{I}$ is infinite and $s_{i}(x)=s_{i}(y)$ for all sufficiently large $i$, or, $\mathcal{I}$ is finite and $s_{i}\left(T^{k}(x)\right)=s_{i}\left(T^{k}(y)\right)$ for all sufficiently large $i$.

(2) Assume that $\tau$ has no coincidences. If there exists $i \in \mathbb{Z}$ such that $x_{i} \neq$ $y_{i}$ and $\left|\mathcal{I}^{+}\left(T^{i}(x)\right)\right|=\infty$ then it is a distal pair. Otherwise the pair is asymptotic. Anyway $\mathbf{L Y}\left(X_{\tau}, T\right)=\emptyset$.

Proof. Let $\pi(x)=\pi(y)=\left(\delta_{i}\right)_{i \in \mathbb{N}}$ and $\mathcal{I}=\left\{i_{0}, i_{1}, \ldots\right\}$, where $i_{j}<i_{j+1}$ for $j \in \mathbb{N}$. We recall we have $\left|s_{i}(x)\right|=\left|s_{i}(y)\right|$ for any $i \in \mathbb{N}$.

(1) First assume that $|\mathcal{I}|=\infty$. Then by Lemma 2.5

$$
x(0, \infty)=x_{0} \tau^{i_{0}}\left(s_{i_{0}}(x)\right) \tau^{i_{1}}\left(s_{i_{1}}(x)\right) \ldots ; y(0, \infty)=y_{0} \tau^{i_{0}}\left(s_{i_{0}}(y)\right) \tau^{i_{1}}\left(s_{i_{1}}(y)\right) \ldots
$$

Since $\tau$ is one-to-one, $(x, y) \in \mathbf{A}\left(X_{\tau}, T\right)$ if and only if $s_{i}(x)=s_{i}(y)$ for any $i \in \mathcal{I}$ large enough. Now assume that $|\mathcal{I}|<\infty$. In this case $\left|\mathcal{I}^{+}\left(T^{k}(x)\right)\right|=\infty$, then by considering $\left(T^{k}(x), T^{k}(y)\right)$ instead of $(x, y)$ in previous arguments we conclude (1). (2) Assume that $\tau$ has no coincidences. If $x_{i} \neq y_{i}$ for some $i \in \mathbb{Z}$ such that $\left|\mathcal{I}^{+}\left(T^{i}(x)\right)\right|=\left|\mathcal{I}^{+}\left(T^{i}(y)\right)\right|=\infty$, then for any $j \in \mathcal{I}^{+}\left(T^{i}(x)\right)$ all corresponding symbols of $s_{j}\left(T^{i}(x)\right)$ and $s_{j}\left(T^{i}(y)\right)$, and consequently of $\tau^{j}\left(s_{j}\left(T^{i}(x)\right)\right)$ and $\tau^{j}\left(s_{j}\left(T^{i}(y)\right)\right)$, are different, which proves that $(x, y)$ is distal. Otherwise there is $i \in \mathbb{Z}$ such that $x_{i}=y_{i}$ and $\left|\mathcal{I}^{+}\left(T^{i}(x)\right)\right|=\left|\mathcal{I}^{+}\left(T^{i}(y)\right)\right|=\infty$. Then, $s_{j}\left(T^{i}(x)\right)=$ $s_{j}\left(T^{i}(y)\right)$ for any $j \in \mathbb{N}$ which implies that $(x, y)$ is asymptotic. This completes the proof of (2).

Let $A$ be an alphabet. In the sequel we will regard $A^{2}$ as an alphabet of letter-pairs. We identify a pair of words $(u, v)$, with $|u|=|v|$, with a word of $A^{2}$ in the obvious way.

The next proposition provides a general criterion for existence of Li-Yorke pairs. 
Proposition 3.5. Let $\tau: A \rightarrow A^{+}$be a one-to-one primitive substitution of constant length $p$ such that $X_{\tau}$ is not finite. Then the system has Li-Yorke pairs if and only if there exist $m \in \mathbb{N}, a, b \in A, a \neq b$, such that $\tau^{m}(a)=u a v, \tau^{m}(b)=u^{\prime} b v^{\prime}$ with $|u|=\left|u^{\prime}\right|,|v|=\left|v^{\prime}\right|, v \neq v^{\prime}$ and $v$ coincides in at least one coordinate with $v^{\prime}$.

Proof. First we prove the condition is necessary. Let $(x, y) \in \mathbf{L Y}\left(X_{\tau}, T\right)$. Since $\pi(x)=\pi(y)$, one has $k^{+}(x)=k^{+}(y)=k$ and $\mathcal{I}^{+}(x)=\mathcal{I}^{+}(y)=\mathcal{I}$. If $|\mathcal{I}|<\infty$ instead of $(x, y)$ consider the pair $\left(T^{k}(x), T^{k}(y)\right)$ which is also a Li-Yorke pair and for which $\left|\mathcal{I}\left(T^{k}(x)\right)\right|=\infty$. So we can assume $|\mathcal{I}|=\infty$; by Lemma 2.5 $x(0, \infty)=$ $x_{0} \tau^{i_{0}}\left(s_{i_{0}}(x)\right) \ldots \tau^{i_{j}}\left(s_{i_{j}}(x)\right) \ldots$ and $y(0, \infty)=y_{0} \tau^{i_{0}}\left(s_{i_{0}}(y)\right) \ldots \tau^{i_{j}}\left(s_{i_{j}}(y)\right) \ldots$, where $i_{0}<$ $i_{1}<\ldots<i_{j}<\ldots$ are the elements of $\mathcal{I}$. Since $(x, y)$ is a Li-Yorke pair the set $\mathcal{J} \subseteq \mathcal{I}, \mathcal{J}=\left\{i \in \mathcal{I} \mid s_{i}(x) \neq s_{i}(y)\right\}$, is infinite. This implies, by (2.1), that for any large enough $i \in \mathbb{N}, x_{0}^{(i)} \neq y_{0}^{(i)}$. There are two cases.

Case 1: $\mathcal{I} \backslash \mathcal{J}$ is infinite.

For any $i \in \mathcal{I} \backslash \mathcal{J}, s_{i}(x)=s_{i}(y)$ so $\tau^{m}\left(s_{i}(x)\right)=\tau^{m}\left(s_{i}(y)\right)$ for any $m \in \mathbb{N}$. Consider large integers $0<i<j<k$ such that $i, k \in \mathcal{I} \backslash \mathcal{J}, j \in \mathcal{J}$ and $x_{0}^{(i)} \neq y_{0}^{(i)}$. Then, by using (2.1) several times, we get

$$
\tau^{k-i+1}\left(x_{0}^{(k+1)}\right)=u x_{0}^{(i)} v \tau^{(j-i)}\left(s_{j}(x)\right) w \tau^{k-i}\left(s_{k}(x)\right)
$$

and

$$
\tau^{k-i+1}\left(y_{0}^{(k+1)}\right)=u^{\prime} y_{0}^{(i)} v^{\prime} \tau^{(j-i)}\left(s_{j}(y)\right) w^{\prime} \tau^{k-i}\left(s_{k}(y)\right)
$$

with $|u|=\left|u^{\prime}\right|,|v|=\left|v^{\prime}\right|$ and $|w|=\left|w^{\prime}\right|$. Then $v \tau^{(j-i)}\left(s_{j}(x)\right) w \tau^{k-i}\left(s_{k}(x)\right)$ and $v^{\prime} \tau^{(j-i)}\left(s_{j}(y)\right) w^{\prime} \tau^{k-i}\left(s_{k}(y)\right)$ are different (because $\left.j \in \mathcal{J}\right)$ and have at least one coincidence (because $k \in \mathcal{I} \backslash \mathcal{J}$ ). Since $\mathcal{J}$ and $\mathcal{I} \backslash \mathcal{J}$ are infinite there exist triples $i<j<k$ as before such that $\left(x_{0}^{(k+1)}, y_{0}^{(k+1)}\right)=\left(x_{0}^{(i)}, y_{0}^{(i)}\right)$. Taking $a=x_{0}^{(k+1)}$, $b=y_{0}^{(k+1)}$ and $m=k-i+1$ one concludes.

Case 2: $\mathcal{I} \backslash \mathcal{J}$ is finite.

One can assume $\mathcal{J}=\mathcal{I}$. Define for any $(a, b) \in A^{2}$ the set

$$
\mathcal{T}_{\tau}(a, b)=\left\{(c, d) \in A^{2}: \exists m \leq|A|^{2}+1,(c, d) \text { occurs in }\left(\tau^{m}(a), \tau^{m}(b)\right)\right\} .
$$

Since $(x, y)$ is a Li-Yorke pair and $s_{i}(x) \neq s_{i}(y)$ for any $i \in \mathcal{I}$, there is an infinite set $\mathcal{R} \subseteq \mathcal{I}$ such that for any $i \in \mathcal{R}$ there exists $0 \leq j<\left|s_{i}(x)\right|$ with $\mathcal{T}_{\tau}\left(\left(s_{i}(x)\right)_{j},\left(s_{i}(y)\right)_{j}\right)$ containing a diagonal pair $(c, c)$. That is, for some integer $0 \leq m \leq|A|^{2}+1$, $\tau^{m}\left(\left(s_{i}(x)\right)_{j}\right)$ and $\tau^{m}\left(\left(s_{i}(y)\right)_{j}\right)$ have a coincidence. Since $s_{i}(x) \neq s_{i}(y)$ we conclude for large $i \in \mathcal{R}$ that $\tau^{m+1}\left(x_{0}^{(i+1)}\right)$ and $\tau^{m+1}\left(y_{0}^{(i+1)}\right)$ have at least one coincidence and two non-coincidences, with one coincidence and one non-coincidence after a non-coincidence. An argument similar to the one used to conclude in Case 1 finishes the proof.

Let us now prove the condition is sufficient. Assume there exist $m \in \mathbb{N}, a, b \in A$, $a \neq b$, such that $\tau^{m}(a)=u a v, \tau^{m}(b)=u^{\prime} b v^{\prime}$, with $|u|=\left|u^{\prime}\right|,|v|=\left|v^{\prime}\right|, v \neq v^{\prime}$ and $v$ coincides in at least one coordinate with $v^{\prime}$. Without loss of generality suppose $m=1$. If $|u|>0$ consider the points of $X_{\tau}$

$$
x=\ldots \tau^{i}(u) \ldots \tau^{2}(u) \tau(u) u . a v \tau(v) \tau^{2}(v) \ldots \tau^{i}(v) \ldots
$$

and

$$
y=\ldots . \tau^{i}\left(u^{\prime}\right) \ldots \tau^{2}\left(u^{\prime}\right) \tau\left(u^{\prime}\right) u^{\prime} . a v^{\prime} \tau\left(v^{\prime}\right) \tau^{2}\left(v^{\prime}\right) \ldots \tau^{i}\left(v^{\prime}\right) \ldots
$$

where the zero coordinate is just after the central dot. Clearly $(x, y)$ is a Li-Yorke pair. If $|u|=0$ taking powers of $\tau$ we can assume there exist $c, d \in A$ such that $c a$ 
and $d b$ are sub-words of $X_{\tau}$ and $\tau(c)=\ldots c, \tau(d)=\ldots d$. In this case consider the points

$$
x=\lim _{k \rightarrow \infty} \tau^{k}(c) . a v \tau(v) \tau^{2}(v) \ldots \tau^{i}(v) \ldots ; y=\lim _{k \rightarrow \infty} \tau^{k}(d) . b v^{\prime} \tau\left(v^{\prime}\right) \tau^{2}\left(v^{\prime}\right) \ldots \tau^{i}\left(v^{\prime}\right) \ldots
$$

which form a Li-Yorke pair.

The following result can be deduced from the proof of Proposition 3.5 .

Corollary 3.6. Let $\tau: A \rightarrow A^{+}$be a one-to-one primitive substitution of constant length $p$ such that $X_{\tau}$ is not finite. Assume $\tau$ has overall coincidences. Let $\{x, y\} \subset$ $X_{\tau}$ be such that $\pi(x)=\pi(y)$. Then $(x, y) \in \mathbf{L Y}\left(X_{\tau}, T\right)$ if and only if for infinitely many $i \in \mathbb{N}$ we have either $s_{i}(x) \neq s_{i}(y)$, or, $s_{i}\left(T^{k^{+}(x)}(x)\right) \neq s_{i}\left(T^{k^{+}(y)}(y)\right)$.

Here are two general sufficient conditions for the existence of $\mathrm{Li}-$ Yorke pairs. The proof is left to the reader.

Corollary 3.7. Let $\tau: A \rightarrow A^{+}$be a one-to-one primitive substitution of constant length $p$ such that $X_{\tau}$ is not finite.

(1) If there exists $m \in \mathbb{N}$ such that for any $a, b \in A, \tau^{m}(a)$ and $\tau^{m}(b)$ have coincidences and at least two non-coincidences, then $\mathbf{L Y}\left(X_{\tau}, T\right) \neq \emptyset$.

(2) Assume there exist $m \in \mathbb{N}$ and $a, b \in A$ such that $\tau^{m}(a)$ and $\tau^{m}(b)$ have coincidences and at least two non-coincidences. If for any $\left(c, c^{\prime}\right),\left(d, d^{\prime}\right) \in$ $A^{2}, c \neq c^{\prime}, d \neq d^{\prime}$, there is $i \in \mathbb{N}$ such that $\left(c, c^{\prime}\right)$ is a letter-pair of $\left(\tau^{i}(d), \tau^{i}\left(d^{\prime}\right)\right)$ then the system has Li-Yorke pairs.

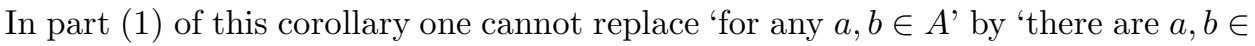
$A^{\prime}$. Consider the substitution $\tau:\{0,1,2,3\} \rightarrow\{0,1,2,3\}^{+}$given by $\tau(0)=0123$, $\tau(1)=1032, \tau(2)=1023$ and $\tau(3)=0132$. For any $m \in \mathbb{N}$ and $a, b \in\{0,1,2,3\}$, either $\tau^{m}(a)$ and $\tau^{m}(b)$ have a common prefix and after this prefix they have no coincidences, or they have a suffix in common but no coincidences before this suffix starts.

Then one proves by contradiction that $\tau$ does not check the condition of Proposition 3.5, and $\mathbf{L Y}\left(X_{\tau}, T\right)=\emptyset$. On the other hand $\tau^{m}(1)$ and $\tau^{m}(2)$ have coincidences and at least two non-coincidences so 1 and 2 verify the condition in part (1) of the previous corollary for any $m \in \mathbb{N}$.

Now we give a necessary and sufficient condition to have uncountably many LiYorke pairs.

Proposition 3.8. Let $\tau: A \rightarrow A^{+}$be a one-to-one primitive substitution of constant length $p$ such that $X_{\tau}$ is not finite. Then the set of orbits of Li-Yorke pairs of $X_{\tau}$ is either finite or uncountable. Moreover, the set of Li-Yorke pairs is uncountable if and only if

$$
\begin{aligned}
& \exists m \in \mathbb{N}, \exists a, b \in A, a \neq b, \tau^{m}(a)=\text { uavaw, } \tau^{m}(b)=u^{\prime} b v^{\prime} b w^{\prime} \\
& \text { with }|u|=\left|u^{\prime}\right|,|v|=\left|v^{\prime}\right|,|w|=\left|w^{\prime}\right|, \text { vaw and } v^{\prime} b w^{\prime} \text { have a coincidence. }
\end{aligned}
$$

Proof. First we prove that (3.1) is a necessary and sufficient condition to have uncountably many Li-Yorke pairs.

Assume (3.1) holds. Without loss of generality suppose that $m=1$. We set $p_{0}=u$, $p_{1}=u a v, s_{0}=v a w, s_{1}=w, q_{0}=u^{\prime}, q_{1}=u^{\prime} b v^{\prime}, t_{0}=v^{\prime} b w^{\prime}$ and $t_{1}=w^{\prime}$. Given a sequence $\underline{\mathbf{n}}=\left(n_{i}\right)_{i \in \mathbb{N}} \in\{0,1\}^{\mathbb{N}}$ which contains infinitely many 0 's and 1's we define points

$$
x(\underline{\mathbf{n}})=\ldots \tau^{2}\left(p_{n_{2}}\right) \tau\left(p_{n_{1}}\right) p_{n_{0}} . a s_{n_{0}} \tau^{1}\left(s_{n_{1}}\right) \tau^{2}\left(s_{n_{2}}\right) \ldots
$$




$$
y(\underline{\mathbf{n}})=\ldots \tau^{2}\left(q_{n_{2}}\right) \tau\left(q_{n_{1}}\right) q_{n_{0}} . b t_{n_{0}} \tau^{1}\left(t_{n_{1}}\right) \tau^{2}\left(t_{n_{2}}\right) \ldots
$$

where the central dot separates negative and positive coordinates. One verifies easily that $x(\underline{\mathbf{n}})$ and $y(\underline{\mathbf{n}})$ belong to $X_{\tau}$ and that they form a Li-Yorke pair. Also if $\underline{\mathbf{n}}, \underline{\mathbf{n}}^{\prime} \in\{0,1\}^{\mathbb{N}}, \underline{\mathbf{n}} \neq \underline{\mathbf{n}}^{\prime}$, then $x(\underline{\mathbf{n}}) \neq x\left(\underline{\mathbf{n}}^{\prime}\right)$ and $y(\underline{\mathbf{n}}) \neq y\left(\underline{\mathbf{n}}^{\prime}\right)$, because they do not have the same image in the odometer. Therefore, $\mathbf{L Y}\left(X_{\tau}, T\right)$ is uncountable.

Now assume that condition (3.1) is not satisfied: then the number of orbits of Li-Yorke pairs in $\mathbf{L Y}\left(X_{\tau}, T\right)$ is finite. To prove this claim we consider a $\mathrm{Li}-$ Yorke pair $(x, y) \in \mathbf{L Y}\left(X_{\tau}, T\right)$ and show that the sequences $\left(x^{(i)}\left(-\delta_{i},-\delta_{i}+p-\right.\right.$ $\left.1), y^{(i)}\left(-\delta_{i},-\delta_{i}+p-1\right)\right)_{i \in \mathbb{N}}$ and $\left(\delta_{i}\right)_{i \in \mathbb{N}}$ are ultimately periodic and periods can be taken to be smaller than $|A|^{2}+1$.

As in the proof of Proposition 3.5 one can assume that $\left|\mathcal{I}^{+}(x)\right|=\left|\mathcal{I}^{+}(y)\right|=\infty$. Therefore, for reasons similar to those in Cases 1 and 2 of the proof of Proposition 3.5. there exist $a, b \in A, a \neq b$, such that for infinitely many $i \in \mathbb{N}$ there is $m_{i} \in \mathbb{N}$ for which

$$
\begin{aligned}
& \text { (i) } x_{0}^{(i)}=x_{0}^{\left(i-m_{i}\right)}=a, y_{0}^{(i)}=y_{0}^{\left(i-m_{i}\right)}=b \\
& \text { (ii) } \tau^{m_{i}}\left(x_{0}^{(i)}\right)=u_{i} x_{0}^{\left(i-m_{i}\right)} v_{i}, \tau^{m_{i}}\left(y_{0}^{(i)}\right)=u_{i}^{\prime} y_{0}^{\left(i-m_{i}\right)} v_{i}^{\prime}, \\
& \quad \text { with }\left|u_{i}\right|=\left|u_{i}^{\prime}\right|,\left|v_{i}\right|=\left|v_{i}^{\prime}\right|, v_{i} \text { has a coincidence with } v_{i}^{\prime} .
\end{aligned}
$$

Claim. For all $r \geq 1$ the word $\left(\tau^{r}(a), \tau^{r}(b)\right)$ contains $(a, b)$ as a sub-word at most once.

Proof of the claim. Assume this is not true. Then for some $r \geq 1, \tau^{r}(a)=$ paqas, $\tau^{r}(b)=p^{\prime} b q^{\prime} b s^{\prime}$, with $|p|=\left|p^{\prime}\right|$ and $|q|=\left|q^{\prime}\right|$. Therefore for any $i \in \mathbb{N}$ such that condition (3.2) holds $\tau^{r+m_{i}}(a)=$ uavaw, $\tau^{r+m_{i}}(b)=u^{\prime} b v^{\prime} b w^{\prime}$, with $|u|=\left|u^{\prime}\right|$, $|v|=\left|v^{\prime}\right|,|w|=\left|w^{\prime}\right|$ and vaw has at least one coincidence with $v^{\prime} b w^{\prime}$. This is a contradiction since condition (3.1) does not hold.

Let $r$ be the smallest positive integer such that $\left(\tau^{r}(a), \tau^{r}(b)\right)$ has $(a, b)$ as a subword. It results from the claim that $(a, b)$ appears exactly once in $\left(\tau^{k r}(a), \tau^{k r}(b)\right)$ for any $k \geq 1$. Moreover, again by the claim, we have that $(a, b)$ is not a sub-word of $\left(\tau^{k r+l}(a), \tau^{k r+l}(b)\right)$ for $k \geq 1$ and $l \in\{1, \ldots, r-1\}$. Since there are infinitely many $i \in \mathbb{N}$ such that condition (3.2) holds, this implies that the sequences $\left(x^{(i)}\left(-\delta_{i},-\delta_{i}+\right.\right.$ $\left.p-1), y^{(i)}\left(-\delta_{i},-\delta_{i}+p-1\right)\right)_{i \in \mathbb{N}}$ and $\left(\delta_{i}\right)_{i \in \mathbb{N}}$ are ultimately periodic, and periods can be taken to be smaller than $|A|^{2}+1$. This proves that the set of Li-Yorke pairs is countable. Moreover, by (2.3) and (2.4),$(x, y)$ are in the orbit of a $\mathrm{Li}-$ Yorke pair $\left(x^{\prime}, y^{\prime}\right)$ such that the sequences $\left(x^{\prime(i)}\left(-\delta_{i},-\delta_{i}+p-1\right)\right)_{i \in \mathbb{N}},\left(y^{\prime(i)}\left(-\delta_{i},-\delta_{i}+p-1\right)\right)_{i \in \mathbb{N}}$, are periodic. To conclude we remark, again by (2.3) and (2.4), that such a sequence determines a finite number of points; thus there are finitely many orbits of Li-Yorke pairs in $X_{\tau} \times X_{\tau}$.

Proposition 3.9. Let $\tau: A \rightarrow A^{+}$be a one-to-one primitive substitution of constant length $p$ such that $X_{\tau}$ is not finite and condition (3.1) holds. Then $\left(X_{\tau}, T\right)$ has strong Li-Yorke pairs.

Proof. By taking a power of the substitution $\tau$ we can assume that $\exists a, b \in A, a \neq b$, $\tau(a)=$ uavaw, $\tau(b)=u^{\prime} b v^{\prime} b w^{\prime}$ with $|u|=\left|u^{\prime}\right| \neq 0,|v|=\left|v^{\prime}\right|,|w|=\left|w^{\prime}\right| \neq 0$, vaw and $v^{\prime} b w^{\prime}$ have a coincidence. Then the points

$$
\begin{gathered}
x=\ldots \tau^{2 m}(u) \tau^{2 m-1}(\text { uav }) \ldots \tau(u a v) u . a v a w \tau(w) \ldots \tau^{2 m-1}(w) \tau^{2 m}(v a w) \ldots \\
y=\ldots \tau^{2 m}\left(u^{\prime}\right) \tau^{2 m-1}\left(u^{\prime} b v^{\prime}\right) \ldots \tau\left(u^{\prime} b v^{\prime}\right) u^{\prime} . b v^{\prime} b w^{\prime} \tau\left(w^{\prime}\right) \ldots \tau^{2 m-1}\left(w^{\prime}\right) \tau^{2 m}\left(v^{\prime} b w^{\prime}\right) \ldots
\end{gathered}
$$


form a Li-Yorke pair which is recurrent. Indeed, for every $n \in \mathbb{N}$ there is $m \in \mathbb{N}$ such that $(x(-n, n), y(-n, n))$ is a sub-word of $\left(\tau^{2 m}(a), \tau^{2 m}(b)\right)$.

We deduce the following equivalences.

Corollary 3.10. Let $\tau: A \rightarrow A^{+}$be a one-to-one primitive substitution of constant length $p$ such that $X_{\tau}$ is not finite. Then the following statements are equivalent.

(1) $\left(X_{\tau}, T\right)$ has uncountably many Li-Yorke pairs;

(2) $\left(X_{\tau}, T\right)$ has infinitely many Li-Yorke orbits;

(3) $\left(X_{\tau}, T\right)$ has at least one strong Li-Yorke pair;

(4) $\left(X_{\tau}, T\right)$ has uncountably many strong Li-Yorke orbits;

(5) Condition (3.1) holds for $\tau$.

Proof. If $\left(X_{\tau}, T\right)$ has finitely many Li-Yorke orbits then $\left(X_{\tau}, T\right)$ has countably many Li-Yorke pairs. Consequently, (1) implies (2).

Proposition 3.9 gives that (2) implies (3).

We use a remark of Akin to prove that (3) implies (4). Let $(x, y)$ be a strong LiYorke pair of $\left(X_{\tau}, T\right)$. It is transitive in the closure of its orbit, $K=\operatorname{clos}(\{(T \times$ $\left.T)^{n}(x, y): n \in \mathbb{Z}\right\}$ ), which intersects the diagonal. Then the set of transitive points of $(K, T \times T)$ is a dense $G_{\delta}$ subset of $K$, therefore uncountable; it is also a set of Li-Yorke pairs of $\left(X_{\tau}, T\right)$. Hence, $\left(X_{\tau}, T\right)$ has uncountably many strong Li-Yorke pairs and, consequently, uncountably many strong Li-Yorke orbits.

Statement (4) implies (5) by Proposition 3.8. In the same way (5) implies (1).

\section{Examples.}

(1) The substitution $\tau:\{a, b, c\} \rightarrow\{a, b, c\}^{+}, \tau(a)=a b a, \tau(b)=b c a, \tau(c)=c c a$, has countably many Li-Yorke pairs. Corollary 3.10 says that $\left(X_{\tau}, T\right)$ has no strong Li-Yorke pairs.

(2) By Proposition 3.8 the substitution $\tau:\{a, b, c, d\} \rightarrow\{a, b, c, d\}^{+}$given by $\tau(a)=b a a c d, \tau(b)=b b b c d, \tau(c)=b c a b a$ and $\tau(d)=b d a b d$ has uncountably many Li-Yorke pairs; by Proposition $3.9\left(X_{\tau}, T\right)$ has strong Li-Yorke pairs. We show it also has non-recurrent Li-Yorke pairs. Consider the points

$$
x=\lim _{m \rightarrow \infty} \tau^{m}(b) \cdot \tau^{m}(c) ; y=\lim _{m \rightarrow \infty} \tau^{m}(b) \cdot \tau^{m}(d) .
$$

By Corollary 3.6. $(x, y)$ is a Li-Yorke pair of $\left(X_{\tau}, T\right)$. But it is not recurrent since for every positive integers $i, m, i<m,\left(\tau^{i}(c), \tau^{i}(d)\right)$ is not a sub-word of $\left(\tau^{m}(c), \tau^{m}(d)\right)$. (3) Let $A=\{0,1\}$ and $\tau: A \rightarrow A^{+}$be a primitive substitution with $X_{\tau}$ not finite. If condition (3.1) holds then all Li-Yorke pairs of $\left(X_{\tau}, T\right)$ are recurrent.

When $A=\{0,1\}$ we can characterize asymptotic, distal and Li-Yorke pairs.

Corollary 3.11. Let $\tau:\{0,1\} \rightarrow\{0,1\}^{+}$be a one-to-one primitive substitution of constant length $p$ having a coincidence and such that $X_{\tau}$ is not finite. Consider $\{x, y\} \subseteq X_{\tau}$ such that $\pi(x)=\pi(y)$.

(1) Assume $\left|\mathcal{I}^{+}(x)\right|=\infty$. If for infinitely many $i \in \mathcal{I}^{+}(x), s_{i}(x) \neq s_{i}(y)$, then $(x, y)$ is a Li-Yorke pair, otherwise $(x, y)$ is an asymptotic pair.

(2) Assume $\left|\mathcal{I}^{+}(x)\right|<\infty$. If $\tau(0)$ and $\tau(1)$ begin by the same letter then $(x, y)$ is an asymptotic pair, otherwise $(x, y)$ can be an asymptotic or a Li-Yorke pair but not a distal pair. 
Proof. It is a consequence of Proposition 3.4 (1) and Corollary 3.6. We only give the proof of (2). Assume $\left|\mathcal{I}^{+}(x)\right|<\infty$ and put $k(x)=k(y)=k$. If $s_{i}\left(T^{k}(x)\right)=$ $s_{i}\left(T^{k}(y)\right)$ for any large $i \in \mathbb{N}$, by Proposition $3.4(1),(x, y)$ is an asymptotic pair. In particular this condition holds whenever $\tau(0)$ and $\tau(1)$ begins by the same letter. If $s_{i}\left(T^{k}(x)\right) \neq s_{i}\left(T^{k}(y)\right)$ for infinitely many $i \in \mathbb{N}$, since $\tau$ has overall coincidences, then by Corollary 3.6 one concludes that $(x, y)$ is a $\mathrm{Li}$-Yorke pair.

Corollary 3.12. Let $\tau:\{0,1\} \rightarrow\{0,1\}^{+}$be a one-to-one primitive substitution of constant length $p$ such that $X_{\tau}$ is not finite. Consider $\{x, y\} \subseteq X_{\tau}$ such that $\pi(x)=\pi(y)$. Then

(1) If there is no coincidence then $\{x, y\}$ is distal whenever there is $i \in \mathbb{N}$ such that $x_{i} \neq y_{i}$ and $\left|\mathcal{I}^{+}\left(T^{i}(x)\right)\right|=\left|\mathcal{I}^{+}\left(T^{i}(y)\right)\right|=\infty$. In any other case the pair is asymptotic.

(2) If there exists a unique non-coincidence then $\{x, y\}$ is asymptotic.

(3) There exist Li-Yorke pairs if and only if there exist coincidences and at least two non-coincidences. Moreover, $(x, y)$ is a Li-Yorke pair if and only if for infinitely many $i \in \mathbb{N}, s_{i}(x) \neq s_{i}(y)$ if $\left|\mathcal{I}^{+}(x)\right|=\infty$, or, $s_{i}\left(T^{k^{+}(x)}(x)\right) \neq$ $s_{i}\left(T^{k^{+}(y)}(y)\right)$ if $\left|\mathcal{I}^{+}(x)\right|<\infty$.

Proof. (1) Immediate by Proposition 3.4 (2) since the alphabet has size two.

(2) First assume that $\left|\mathcal{I}^{+}(x)\right|=\left|\mathcal{I}^{+}(y)\right|=\infty$. If $x^{(i)}(0)=y^{(i)}(0)$ for any $i \in \mathbb{N}$ then $(x, y)$ is an asymptotic pair. Otherwise for any large enough $i \in \mathcal{I}^{+}(x)$ one has $x^{(i)}(0) \neq y^{(i)}(0)$; thus, since $\tau$ has a unique noncoincidence, $s_{i}(x)=s_{i}(y)$. Then by Proposition $3.4(1) x$ and $y$ are asymptotic. If $\left|\mathcal{I}^{+}(x)\right|=\left|\mathcal{I}^{+}(y)\right|<\infty$ then $\left|\mathcal{I}^{+}\left(T^{k}(x)\right)\right|=\left|\mathcal{I}^{+}\left(T^{k}(y)\right)\right|=\infty$ where $k^{+}(x)=k^{+}(y)=k$. By the argument above $T^{k}(x)$ and $T^{k}(y)$ are asymptotic, and consequently $x$ and $y$ too.

(3) This is a consequence of Corollary 3.6 and Proposition 3.5.

Examples. The system defined by the substitution $\tau:\{0,1\} \rightarrow\{0,1\}^{+}, \tau(0)=$ 010, $\tau(1)=100$ has Li-Yorke pairs and all scrambled sets are finite; the system defined by the Morse substitution $\tau:\{0,1\} \rightarrow\{0,1\}^{+}, \tau(0)=01, \tau(1)=10$ has only distal and asymptotic pairs in the fibres over the odometer; and the system defined by $\tau:\{0,1\} \rightarrow\{0,1\}^{+}, \tau(0)=01, \tau(1)=00$ has only asymptotic pairs in fibres over the odometer. The last substitution defines a Toeplitz subshift.

By increasing the alphabet it is easy to obtain substitution systems with scrambled sets having exactly the cardinality of the alphabet. For $A=\{0, \ldots, n\}, n>0$, consider the primitive one-to-one substitution $\tau: A \rightarrow A^{+}$defined for $a \in A$ by $\tau(a)=0 a a(a+1) 0$ where $n+1=0$. Clearly $X_{\tau}$ is not finite. Define for $a \in A$

$$
x(a)=\ldots \tau^{i}(0) \ldots \tau(0) 0 . a a(a+1) 0 \tau(a(a+1) 0) \ldots \tau^{i}(a(a+1) 0) \ldots \in X_{\tau}
$$

where the zero coordinate is the one just after the central dot. Then $S=\{x(a)$ : $a \in A\}$ is a scrambled set of size $|A|$.

\section{A dynamical System With COUntable SCRAmbled Sets}

Let $n \geq 1$. For $A_{n}=\{0, \ldots, n\}$ define the primitive substitution $\tau_{n}: A_{n} \rightarrow A_{n}^{3}$ by $\tau_{n}(a)=a 0(a+1)$ if $a \neq n$ and $\tau_{n}(n)=n 0 n$. Set $X_{\tau_{n}}=X_{n}$; the shift map on $X_{n}$ is denoted by $T_{n}$. We verify that $X_{n}$ is not finite. Let $\rho_{n}: A_{n+1} \rightarrow A_{n}$ be defined 
by $\rho_{n}(a)=a$ if $a \neq n+1$ and $\rho_{n}(n+1)=n$; obviously $\rho_{n} \circ \tau_{n+1}=\tau_{n} \circ \rho_{n}$. The map $\rho_{n}$ extends by concatenation to any word of $A_{n+1}^{+}$; it induces a factor map that we also call $\rho_{n}: X_{n+1} \rightarrow X_{n}$. Remark that each $\tau_{n}$ satisfies the conditions of Proposition 3.5

Let $(X, T)$ be the minimal dynamical system defined by

$$
X=\left\{\left(x_{n}\right)_{n \geq 1} \in \prod_{n \geq 1} X_{n}: \forall n \geq 1, \rho_{n}\left(x_{n+1}\right)=x_{n}\right\} ;
$$

put $T\left(\left(x_{n}\right)_{n \geq 1}\right)=\left(T_{n}\left(x_{n}\right)\right)_{n \geq 1}$. Each system $\left(X_{n}, T_{n}\right)$ is a factor of $(X, T)$ and the maximal equicontinuous factor of $(X, T)$ and $\left(X_{n}, T_{n}\right)$ is the 3-odometer (see Q ). Denote by $\pi_{n}: X_{n} \rightarrow \mathcal{O}_{3}$ and $\pi: X \rightarrow \mathcal{O}_{3}$ the corresponding factor maps. If $x=\left(x_{n}\right)_{n \geq 1} \in X$ then $\pi_{n}\left(x_{n}\right)=\pi(x)$ for all $n$. Each $\tau_{n}$ has overall coincidences, then $\pi_{n}$ is a proximal map. The inverse limit of proximal maps is a proximal map, so, once again, the Li-Yorke pairs are exactly the non-asymptotic pairs in the fibers of $\pi$. We show that fibres of $\pi$ are at most countable and construct a countable scrambled set. Since any scrambled set is included in one fibre, all scrambled sets of $(X, T)$ are finite or countable.

For any $z \in X_{m}, m \geq 1$, let $\left(z^{(i)}\right)_{i \in \mathbb{N}}$ be the sequence in $X_{m}$ given by Theorem 2.4 Recall that if $\pi_{m}(z)=\left(\delta_{i}\right)_{i \in \mathbb{N}}$ one has

$$
\tau_{m}\left(z_{0}^{(i+1)}\right)=z^{(i)}\left(-\delta_{i},-\delta_{i}+2\right)
$$

and thus

$$
z^{(j)}\left(-\delta_{j},-\delta_{j}+2\right), j<i \text {, are determined once we know } z^{(i)}\left(-\delta_{i},-\delta_{i}+2\right) .
$$

Both properties are extensively used in the proofs of this section.

Lemma 4.1. Let $x \in X_{n}$ for some given $n \geq 1$.

(1) If for infinitely many $i \in \mathbb{N}, x^{(i)}\left(-\delta_{i},-\delta_{i}+2\right)=a_{i} 0\left(a_{i}+1\right)$ for some $a_{i} \in A_{n-1}$, then there is a sequence $v \in\left(A_{n+1}^{3}\right)^{\mathbb{N}}$ such that $\left(y^{(i)}\left(-\delta_{i},-\delta_{i}+\right.\right.$ $2))_{i \in \mathbb{N}}=v$ for any $y \in \rho_{n}^{-1}(\{x\})$. Moreover, for infinitely many $i \in \mathbb{N}$, $v_{i}=a_{i} 0\left(a_{i}+1\right)$ for some $a_{i} \in A_{n}$.

(2) If for any big enough $i \in \mathbb{N}, x^{(i)}\left(-\delta_{i},-\delta_{i}+2\right)=n 0 n$, then there are two sequences $v, v^{\prime} \in\left(A_{n+1}^{3}\right)^{\mathbb{N}}$ with $v_{i}=(n 0(n+1))$ and $v_{i}^{\prime}=((n+1) 0(n+1))$ for any enough large $i \in \mathbb{N}$, such that $\left(y^{(i)}\left(-\delta_{i},-\delta_{i}+2\right)\right)_{i \in \mathbb{N}}=v$ or $v^{\prime}$ for any $y \in \rho_{n}^{-1}(\{x\})$. Moreover, if for infinitely many $i \in \mathbb{N}, \delta_{i}=2$, then $\left(y^{(i)}\left(-\delta_{i},-\delta_{i}+2\right)\right)_{i \in \mathbb{N}}=v^{\prime}$ for any $y \in \rho_{n}^{-1}(\{x\})$.

Proof. Let $y \in X_{n+1}$ and $x=\rho_{n}(y) \in X_{n}$. Put $\pi_{n}(x)=\pi_{n+1}(y)=\left(\delta_{i}\right)_{i \in \mathbb{N}}$, then $\rho_{n}\left(y^{(i)}\left(-\delta_{i},-\delta_{i}+2\right)\right)=x^{(i)}\left(-\delta_{i},-\delta_{i}+2\right)$ for all $i \in \mathbb{N}$.

(1) If $x^{(i)}\left(-\delta_{i},-\delta_{i}+2\right)=a 0(a+1)$ with $a \in A_{n-1}$ then $a 0(a+1)$ is the unique subword of $X_{n+1}$ such that $\rho_{n}(a 0(a+1))=a 0(a+1)$, then $y^{(i)}\left(-\delta_{i},-\delta_{i}+2\right)=a 0(a+1)$. Therefore, if for infinitely many $i \in \mathbb{N}, x^{(i)}\left(-\delta_{i},-\delta_{i}+2\right)=a 0(a+1)$ for some $a \in A_{n-1}$, by (4.2), the sequence $\left(y^{(i)}\left(-\delta_{i},-\delta_{i}+2\right)\right)_{i \in \mathbb{N}}$ is completely determined given $x$, it is unique and for infinitely many $i \in \mathbb{N}, y^{(i)}\left(-\delta_{i},-\delta_{i}+2\right)=a 0(a+1)$ for some $a \in A_{n-1} \subseteq A_{n}$. This completes the proof of (1).

(2) Assume that $x^{(i)}\left(-\delta_{i},-\delta_{i}+2\right)=n 0 n$ for any $i \geq i_{0}$. As $n$ occurs only in the first and last positions of $\tau_{n}(n)$, so $\delta_{i}$ can only be 0 or 2 for $i>i_{0}$. Also, if $x^{(i)}\left(-\delta_{i},-\delta_{i}+2\right)=n 0 n$ then $n 0(n+1)$ and $(n+1) 0(n+1)$ are the only sub-words 
of $X_{n+1}$ such that $\rho_{n}(n 0(n+1))=\rho_{n}((n+1) 0(n+1))=n 0 n$, then for $i \geq i_{0}$, $y^{(i)}\left(-\delta_{i},-\delta_{i}+2\right)=n 0(n+1)$ or $(n+1) 0(n+1)$.

Assume that for some $i \geq i_{0}, y^{(i)}\left(-\delta_{i},-\delta_{i}+2\right)=n 0(n+1)$. Then, by definition of $\tau_{n+1}, y^{(i+1)}\left(-\delta_{i+1},-\delta_{i+1}+2\right)=n 0(n+1)$ and $\delta_{i+1}=0$. Inductively we deduce that $y^{(j)}\left(-\delta_{j},-\delta_{j}+2\right)=n 0(n+1)$ and $\delta_{j}=0$ for any $j>i$. By using (4.2), we conclude $\left(y^{(i)}\left(-\delta_{i},-\delta_{i}+2\right)\right)_{i \in \mathbb{N}}$ can be equal to two possibles sequences. One is such that $\left(y^{(i)}\left(-\delta_{i},-\delta_{i}+2\right)\right)_{i \geq j_{0}}=(n 0(n+1))_{i \geq j_{0}}$ and the other verifies $\left(y^{(i)}\left(-\delta_{i},-\delta_{i}+\right.\right.$ $2))_{i \geq j_{0}}=((n+1) 0(n+1))_{i \geq j_{0}}$, where $j_{0} \in \mathbb{N}$. But, if for infinitely many $i \in \mathbb{N}$, $\delta_{i}=2$, the sequence $\left(y^{(i)}\left(-\delta_{i},-\delta_{i}+2\right)\right)_{i \in \mathbb{N}}$ can take a unique value such that $\left(y^{(i)}\left(-\delta_{i},-\delta_{i}+2\right)\right)_{i \geq j_{0}}=((n+1) 0(n+1))_{i \geq j_{0}}$ for some $j_{0} \in \mathbb{N}$.

Lemma 4.2. Let $x \in X_{n}$ for some given $n \geq 1$.

(1) $\rho_{n}^{-1}(\{x\})$ contains at most two elements; when it contains two, one of them has a unique preimage under $\rho_{n+1}$.

(2) If $x$ has a unique preimage under $\rho_{n}$, there exists a unique sequence $\left(x_{m}\right)_{m \geq n}$ such that $x=x_{n}, x_{m} \in X_{m}$ and $\rho_{m}\left(x_{m+1}\right)=x_{m}$ for $m \geq n$.

Proof. Let $y \in X_{n+1}$ and $x=\rho_{n}(y) \in X_{n}$. Put $\pi_{n}(x)=\pi_{n+1}(y)=\left(\delta_{i}\right)_{i \in \mathbb{N}}$.

(1) We consider three cases.

(a) First suppose that $\delta_{i}=0$ for $i \geq i_{0}$. By definition of $\tau_{n}$ and $\tau_{n+1}, x_{-1}^{(i)}=n$ and $y_{-1}^{(i)}=n+1$ for $i \geq i_{0}$. Then, we deduce from (2.4) and Lemma 2.5 that $x=\lim _{m \rightarrow \infty} \tau_{n}^{m}(n) x(M, \infty)$ and $y=\lim _{m \rightarrow \infty} \tau_{n+1}^{m}(n) y(M, \infty)$ for some $M \in \mathbb{Z}$, where $x(M, \infty), y(M, \infty)$, are uniquely determined by $\left(x^{(i)}\left(-\delta_{i},-\delta_{i}+2\right)\right)_{i \in \mathbb{N}}$ and $\left(y^{(i)}\left(-\delta_{i},-\delta_{i}+2\right)\right)_{i \in \mathbb{N}}$ respectively. Therefore, by Lemma 4.1] $x$ has at most two preimages under $\rho_{n}$, one of which has a unique preimage under $\rho_{n+1}$ since it verifies condition (1) in Lemma 4.1 .

(b) When $\delta_{i}=2$ for $i \geq i_{0}$, by definition of $\tau_{n}$, one has $x^{(i)}\left(-\delta_{i},-\delta_{i}+2\right)=n 0 n$ for $i \geq i_{0}$. We also deduce from (2.3) and Lemma 2.5 that for some $M \in \mathbb{N}$ and $a \in$ $A_{n}, x=x(-\infty, M) \lim _{m \rightarrow \infty} \tau_{n}^{m}(a)$. Therefore $y=y(-\infty, M) \lim _{m \rightarrow \infty} \tau_{n+1}^{m}(a)$ if $a \neq n$, and $y=y(-\infty, M) \lim _{m \rightarrow \infty} \tau_{n+1}^{m}(n)$ or $y=y(-\infty, M) \lim _{m \rightarrow \infty} \tau_{n+1}^{m}(n+1)$ if $a=n$. In both cases $y(-\infty, M)$ is uniquely determined from the sequence $\left(y^{(i)}\left(-\delta_{i},-\delta_{i}+2\right)\right)_{i \in \mathbb{N}}$. But in the case we are studying, by Lemma 4.1 (2), this sequence is uniquely determined from $x$ and verifies $\left(y^{(i)}\left(-\delta_{i},-\delta_{i}+2\right)\right)_{i \geq j_{0}}=((n+$ 1) $0(n+1))_{i \geq j_{0}}$ for some $j_{0} \in \mathbb{N}$. We conclude $y$ can take at most two possible values, which proves the first statement of (1). To finish the proof of (1) in this case observe that if $y=y(-\infty, M) \lim _{m \rightarrow \infty} \tau_{n+1}^{m}(n)$ then $z=z(-\infty, M) \lim _{m \rightarrow \infty} \tau_{n+2}^{m}(n)$ is the unique preimage of $y$ under $\rho_{n+1}$, where $z(-\infty, M)$ is uniquely determined given $y$ by Lemma 4.1 (2).

(c) Finally if none of the two conditions above is checked, $x$ and $y$ are uniquely determined by the sequences $\left(x^{(i)}\left(-\delta_{i},-\delta_{i}+2\right)\right)_{i \in \mathbb{N}}$ and $\left(y^{(i)}\left(-\delta_{i},-\delta_{i}+2\right)\right)_{i \in \mathbb{N}}$. By Lemma 4.1 (2), $y$ can take at most two values, one of which must have a unique preimage under $\rho_{n+1}$ by the same lemma. This completes the proof of (1).

(2) The proof above shows that $y$ is the unique preimage of $x$ under $\rho_{n}$ if and only if one of the following three cases occur:

(i) for infinitely many $i \in \mathbb{N}, x^{(i)}\left(-\delta_{i},-\delta_{i}+2\right)=y^{(i)}\left(-\delta_{i},-\delta_{i}+2\right)=a 0(a+1), a \in$ $A_{n-1}$ and $\delta_{i} \neq 2$; 
(ii) for infinitely many $i \in \mathbb{N}, \delta_{i}=2$, for infinitely many $i \in \mathbb{N}, \delta_{i}=0$ and there is $i_{0} \in \mathbb{N}$ such that $x^{(i)}\left(-\delta_{i},-\delta_{i}+2\right)=n 0 n, y^{(i)}\left(-\delta_{i},-\delta_{i}+2\right)=(n+1) 0(n+1)$ for $i \geq i_{0}$

(iii) there is $i_{0} \in \mathbb{N}$ such that for $i \geq i_{0}, \delta_{i}=2, x^{(i)}\left(-\delta_{i},-\delta_{i}+2\right)=n 0 n$, $y^{(i)}\left(-\delta_{i},-\delta_{i}+2\right)=(n+1) 0(n+1)$ and for some $M \in \mathbb{N}$ and $a \in A_{n-1}, y=$ $y(-\infty, M) \lim _{m \rightarrow \infty} \tau_{n+1}^{m}(a)$.

In case (i), by Lemma 4.1 and the proof of (a) and (c) above, there is a unique $z \in X_{n+2}$ such that $\rho_{n+1}(z)=y$; it is uniquely determined by $\left(y^{(i)}\left(-\delta_{i},-\delta_{i}+2\right)\right)_{i \in \mathbb{N}}$. Moreover, $\left(z^{(i)}\left(-\delta_{i},-\delta_{i}+2\right)\right)_{i \in \mathbb{N}}$ also verifies condition (i). Then we conclude by induction.

In cases (ii) and (iii) the proof is analogous.

Proposition 4.3. The map $\pi: X \rightarrow \mathcal{O}_{3}$ is at most countable-to-one.

Proof. Let $\delta \in \mathbb{Z}_{3}^{\mathbb{N}}$. One has

$$
\pi^{-1}(\{\delta\})=\left\{\left(x_{i}\right)_{i \geq 1} \in X: \pi_{1}\left(x_{1}\right)=\delta\right\}=\cup_{x \in \pi_{1}^{-1}(\{\delta\})} X(x),
$$

where $X(x)=\left\{\left(x_{i}\right)_{i \geq 1} \in X: x_{1}=x\right\}$. By Lemma 4.2 the set $X(x)$ is at most countable; $\pi_{1}^{-1}(\{\delta\})$ is finite by Lemma 2.7 .

Now we construct a countable scrambled set. Let $n \geq 1$ and $m \geq n$. Remark that the words $m(n-1)$ and $n n$ can be obtained as sub-words of $\tau_{m}^{m}(0(n-1))$ and $\tau_{m}^{n}(0 n)$ respectively, then they are sub-words of $X_{m}$. So we can define

$$
x(n)=\lim _{k \rightarrow \infty} \tau_{n}^{k}(n) . n 0 n \tau_{n}(0 n) \ldots \tau_{n}^{i}(0 n) \ldots \in X_{n}
$$

and

$$
y(m, n)=\lim _{k \rightarrow \infty} \tau_{m}^{k}(m) .(n-1) 0 n \tau_{m}(0 n) \ldots \tau_{m}^{i}(0 n) \ldots \in X_{m}
$$

where the symbol after the dot is the zero coordinate. Clearly $\rho_{n}(x(n+1))=$ $\rho_{n}(y(n+1, n+1))=x(n)$ and $\rho_{m}(y(m+1, n))=y(m, n)$, so that

$$
S=\cup_{n \geq 2}\{(x(1), \ldots, x(n-1), y(n, n), y(n+1, n), y(n+2, n), \ldots)\} \subset X .
$$

Proposition 4.4. The countable set $S$ is a scrambled set of $(X, T)$.

Proof. It is enough to observe that $(x(n), y(n, n)) \in \mathbf{A}(X, T),(x(m), y(m, n)) \in$ $\mathbf{L Y}(X, T), m>n$, and $(y(m, n), y(m, k)) \in \mathbf{L Y}(X, T), m \geq n, m \geq k, m \neq k$.

\section{A further EXAmple}

Like the example in $[\mathrm{Au}]$, p.26, and examples in Section 3 the system we describe now is semi-distal but not almost distal; unlike these examples, it is an extension of an irrational rotation and has countably many Li-Yorke pairs.

Let us denote by $\mathbb{S}$ the circle and consider its representation as the quotient group $\mathbb{R} / \mathbb{Z}$. Given a real number $t$ we denote by $\{t\}$ the fractional part of $t$ (that is, $\{t\}$ is the unique representative in $[0,1)$ of the $\bmod \mathbb{Z}$ congruence class of $t$ ). Fix an irrational number $\alpha \in[0,1)$ and consider $R_{\alpha}: \mathbb{S} \rightarrow \mathbb{S}$ to be the rotation by $\alpha$ on the circle, $R_{\alpha}(t)=\{t+\alpha\}$. The system $\left(\mathbb{S}, R_{\alpha}\right)$ is minimal and isometric, and so is distal.

Let $n_{0}=1$. Choose an increasing sequence of positive integers, $\left\{n_{i}: i \geq 1\right\}$, such that the fractional parts $\left\{\left\{n_{j} \alpha\right\}: j \geq 1\right\}$ form a decreasing sequence in the unit interval which converges to $\alpha$. Observe that $\left\{n_{j} \alpha\right\}$ is the fractional part of $R_{\alpha}^{n_{j}}(0)=R_{\alpha}^{n_{j}-1}(\alpha)$. Now choose $\beta^{1}$ so that $\left\{n_{1} \alpha\right\}<\beta^{1}<1$ and $\beta^{1}$ is not a 
rational linear combination of 1 and $\alpha$. In particular, $\beta^{1}+\mathbb{Z}$ is not in the orbit of $\alpha+\mathbb{Z}$ in $\mathbb{S}$. Inductively for $j>1$, choose $\beta^{j}$ rationally independent of $1, \alpha$ and $\beta^{k}$ for $k<j$ such that $\left\{n_{j} \alpha\right\}<\beta^{j}<\left\{n_{j-1} \alpha\right\}$. Let $I_{j}=\left[\left\{n_{j} \alpha\right\}, \beta^{j}\right]$ for $j \geq 1$ and let $K=\{\alpha\} \cup \bigcup_{j \geq 1} I_{j}$. Thus, $K$ is a closed set in the unit interval, and by projecting we can regard it as a closed set in $\mathbb{S}$. The boundary of $K$ consists of $\partial_{\alpha}=\left\{\left\{n_{j} \alpha\right\}: j \geq 0\right\}$ and $\partial_{\beta}=\left\{\beta^{j}: j \geq 1\right\}$. The points of $\partial_{\alpha}$ lie on the orbit of $\alpha$ while the points in $\partial_{\beta}$ are all on distinct orbits different from the $\alpha$ orbit as well. Put $A=\{0,1\}$ and let $c_{0}: \mathbb{S} \rightarrow A$ be the characteristic function of $K$. That is, $c_{0}(t)=1$ if $t \in K$ and $c_{0}(t)=0$ otherwise. Notice that $c_{0}$ is continuous at every point of $\mathbb{S}$ except for those on the boundary of $K$. Let $c_{i}=c_{0} \circ R_{\alpha}^{i}$ for $i \in \mathbb{Z}$. Finally, define $c: \mathbb{S} \rightarrow A^{\mathbb{Z}}$ by $c(t)=\left(c_{i}(t): i \in \mathbb{Z}\right)$. Notice that $c$ defines a commuting map between $\left(\mathbb{S}, R_{\alpha}\right)$ and $\left(A^{\mathbb{Z}}, T\right)$ where $T$ is the shift map. Denote by $\operatorname{cont}(c)$ the continuity set of $c$. It is given by

$$
\operatorname{cont}(c)^{c}=\{\{\alpha+n \alpha\}: n \in \mathbb{Z}\} \cup \bigcup_{j \geq 1}\left\{\left\{\beta^{j}+n \alpha\right\}: n \in \mathbb{Z}\right\} .
$$

The graph of $c, G(c)=\{(t, c(t)): t \in \mathbb{S}\}$, is an $R_{\alpha} \times T$ invariant subset of $\mathbb{S} \times A^{\mathbb{Z}}$. Since $c$ is not continuous, it is not a closed subset. However, $\operatorname{Gcont}(c)=\{(t, c(t))$ : $t \in \operatorname{cont}(c)\}$ form a dense subset of $G(c)$.

Let $Z$ denote the closure of $G(c)$ in $\mathbb{S} \times A^{\mathbb{Z}}$. This is a $R_{\alpha} \times T$ invariant subset of $\mathbb{S} \times A^{\mathbb{Z}}$. We denote by $F: Z \rightarrow Z$ the restriction of $R_{\alpha} \times T$ to $Z$ and by $\rho: Z \rightarrow \mathbb{S}$ the coordinate projection. Clearly, $\rho$ is a factor map from $(Z, F)$ to $\left(\mathbb{S}, R_{\alpha}\right)$. For $t \in \mathbb{S}$ let $Z_{t}=\{(s, c(s)) \in Z: \rho(s)=t\}$ denote the fiber $\rho^{-1}(\{t\})$ in $Z$.

Lemma 5.1. If $t \in \operatorname{cont}(c)$ then $(t, c(t))$ is the unique point of $Z$ such that $\rho(t, c(t))=t$. In particular, $Z_{t}$ is a singleton set. More generally, if $(t, x) \in Z$ and $t$ is a continuity point for $c_{i}$ (that is, $R_{\alpha}^{i}(t)$ is not in the boundary of $K$ ) then $x_{i}=c_{i}(t)$.

Proof. We leave it as an exercise.

As we observed before the set $G \operatorname{cont}(c)$ is dense in $Z$, then $\rho$ is an almost one-toone map. Such maps are always proximal and minimality is preserved by almost one-to-one lifts. Therefore $(Z, F)$ is a minimal system.

For each $t \in \operatorname{cont}(c)$ there is a unique $F$ orbit in $Z$ lying over its $R_{\alpha}$ orbit in $\mathbb{S}$. The remaining orbits are those of the $\beta^{j}$ for $j \geq 1$ and the orbit of $\alpha$.

Lemma 5.2. For $j \geq 1$ the fiber $Z_{\beta^{j}}$ consists of two points $z^{j+}=\left(\beta^{j}, a^{j}\right)$ and $z^{j-}=\left(\beta^{j}, b^{j}\right)$ with $a_{i}^{j}=b_{i}^{j}=c_{i}\left(\beta^{j}\right)$ for all $i \geq 1$ and with $a_{0}^{j}=1$ and $b_{0}^{j}=0$.

Proof. Let $j \geq 1$. If a sequence $\left(t_{k}\right)_{k \in \mathbb{N}}$ approaches $\beta^{j}$ from above then, eventually, $t_{k} \notin K$ and so, eventually, $c_{0}\left(t_{k}\right)=0$. For $i \geq 1$ the function $c_{i}$ is continuous at $\beta^{j}$, so the sequence $\left(c_{i}\left(t_{k}\right)\right)_{k \in \mathbb{N}}$ has limit $c_{i}\left(\beta^{j}\right)$. Thus, $\left(\left(t_{k}, c\left(t_{k}\right)\right)\right)_{k \in \mathbb{N}}$ approaches $z^{j-}$. If $\left(t_{k}\right)_{k \in \mathbb{N}}$ approaches $\beta^{j}$ from below then, eventually, $t_{k} \in K$, and so $\left(\left(t_{k}, c\left(t_{k}\right)\right)\right)_{k \in \mathbb{N}}$ approaches $z^{j+}$. Any real sequence admits a monotone subsequence, then these are the only points in $Z_{\beta^{j}}$.

Lemma 5.3. The fiber $Z_{\alpha}$ consists of three points $z^{+1}=(\alpha, a), z^{-}=(\alpha, b)$ and $z^{+0}=(\alpha, \bar{a})$ such that:

$$
\text { for } i \notin\left\{n_{j}-1: j \geq 0\right\}, a_{i}=\bar{a}_{i}=b_{i}=c_{i}(\alpha)
$$




$$
\begin{gathered}
\text { for } i \in\left\{n_{j}-1: j>0\right\}, a_{i}=\bar{a}_{i}=1, b_{i}=0 \\
\text { for } i=0, a_{0}=1, \bar{a}_{0}=b_{0}=0 .
\end{gathered}
$$

Proof. If $i \notin\left\{n_{0}-1, n_{1}-1, \ldots\right\}$ then $c_{i}$ is continuous at $\alpha$. If a sequence $\left(t_{k}\right)_{k \in \mathbb{N}}$ approaches $\alpha$ then $\left(c_{i}\left(t_{k}\right)\right)_{k \in \mathbb{N}}$ approaches $c_{i}(\alpha)$ for such $i$. If $\left(t_{k}\right)_{k \in \mathbb{N}}$ approaches $\alpha$ from below then it lies in the complement of $K$ and so $c_{0}\left(t_{k}\right)=0$ for all $k \in \mathbb{N}$. For $i=n_{j}-1$ with $j>0$ the fractional part of $\left(R_{\alpha}^{n_{j}-1}\left(t_{k}\right)\right)_{k \in \mathbb{N}}$ approaches $\left\{n_{j} \alpha\right\}$ from below and so the rotated sequence is also eventually in the complement of $K$. Hence $\left(\left(t_{k}, c\left(t_{k}\right)\right)\right)_{k \in \mathbb{N}}$ approaches $z^{-}$. If $\left(t_{k}\right)_{k \in \mathbb{N}}$ approaches $\alpha$ from above then for $i=n_{j}-1$ with $j>0$ the sequence of the fractional parts of $R_{\alpha}^{n_{j}-1}\left(t_{k}\right), k \in \mathbb{N}$, approaches $\left\{n_{j} \alpha\right\}$ from above and so the rotated sequence eventually enters the interval $I_{j}$. Hence, the limit of $\left(c_{i}\left(t_{k}\right)\right)_{k \in \mathbb{N}}$ is 1 for such $i$ 's. Finally, for $i=0$, observe that $\alpha$ is a limit point from above of points in $K$ and of points in $\mathbb{S} \backslash K$. If we choose a sequence $\left(t_{k}\right)_{k \in \mathbb{N}}$ with $t_{k} \in K$ for all $k \in \mathbb{N}$ then $c_{0}\left(t_{k}\right)=1$ and the sequence $\left(\left(t_{k}, c\left(t_{k}\right)\right)\right)_{k \in \mathbb{N}}$ approaches $z^{+1}$. If instead we choose a sequence $\left(t_{k}\right)_{k \in \mathbb{N}}$ with $t_{k} \notin K$ for all $k \in \mathbb{N}$ then $c_{0}\left(t_{k}\right)=0$ and $\left(\left(t_{k}, c\left(t_{k}\right)\right)\right)_{k \in \mathbb{N}}$ approaches $z^{+0}$. Any sequence approaching $\alpha$ has a subsequence of one of these three types. Hence, the fiber consists of the three points specified in the statement.

Since $\left(\mathbb{S}, R_{\alpha}\right)$ is a distal system, a proximal pair of distinct points in $Z$ must lie in the same fiber of $\rho$.

It is clear that the pair of orbits which lie over the orbit of $\beta^{j}$ is an asymptotic pair for each $j \geq 1$. There remain the three orbits which lie over the orbit of $\alpha$. Obviously, the pair $\left(z^{+0}, z^{+1}\right)$ is an asymptotic pair, while the pairs $\left(z^{+0}, z^{-}\right)$and $\left(z^{+1}, z^{-}\right)$are proximal pairs, but not asymptotic. Thus, the Li-Yorke set is the countable collection consisting of the orbits of these two pairs.

Now by Akin's remark (see the proof of Corollary 3.10) and the last discussion we conclude that $(Z, T \times \sigma)$ contains no strong Li-Yorke pairs.

Acknowledgments. We thank Pierre Arnoux and Xiangdong Ye for fruitful discussions and comments. We also thank all the valuable comments and suggestions of the anonymous referees which in particular help to give the final form to the example in Section 5. The authors acknowledge financial support from Nucleus Millennium Information and Randomness P01-005, FONDECYT 1010447 and ECOS-Conicyt cooperation agreement C99E10.

\section{REFERENCES}

[AA] E. Akin, J. Auslander, Distality concepts for Ellis actions. Preprint.

$[\mathrm{Au}] \quad$ J. Auslander, Minimal flows and their extensions, Mathematic Studies 153, North Holland, 1988.

[BDH] M. Barge, B. Diamond, C. Holton, Asymptotic orbits of primitive substitutions, Theoret. Comput. Sci. 301 (2003), 439-450.

[BGKM] F. Blanchard, E. Glasner, S. Kolyada, A. Maass, On Li-Yorke pairs, J. Reine Angew. Math. 547, 51-68 (2002).

[De] F.M. Dekking, The spectrum of dynamical systems arising from substitutions of constant length, Z. Wahrscheinlichkeitstheorie und Verw. Gebiete 41, 221-239 (1977/1978).

[HL] Harju, T., Linna, M., On the periodicity of morphisms on free monoids, RAIRO Inform. Théor. Appl. 20 (1986), 47-54.

[HZ] C. Holton, L. Zamboni, Directed graphs and substitutions, Theory Comput. Syst. 34, 545-564 (2001). 
[HY] W. Huang, XD. Ye, Devaney's chaos or 2-scattering implies Li-Yorke chaos, Topology Appl. 117, 259-272 (2002).

[LY] T.Y. Li, J.A. Yorke, Period three implies chaos, Amer. Math. Monthly 82, 985-992 (1975).

[MS] F. Mignosi, P. Séébold, If a DOL-language is $k$-power free then it is circular, ICALP 1993, Lect. Notes in Comp. Sci. 700.

[Mo] B. Mossé, Reconnaissabilité des substitutions et complexité des suites automatiques, Bull. Soc. Math. France 124, 329-346 (1996).

[Pa] Pansiot, J.-J., Decidability of periodicity for infinite words, RAIRO Inform. Théor. Appl. 20 (1986), 43-46.

[Q] M. Queffélec, Substitution Dynamical systems-spectral analysis, Lecture Notes in Mathematics 1294, Springer-Verlag, Berlin, 1987.

[Y] X.D. Ye, personal communication.

Institut de Mathématiques de Luminy (UPR 9016 du CNRS, FRUMAM) ; Case 907, 163 avenue de Luminy, 13288 Marseille Cedex 09, France

E-mail address: blanchar@iml.univ-mrs.fr

Laboratoire Amiénois de Mathématiques Fondamentales et Appliquées, CNRS-UMR 6140, Université de Picardie Jules Verne, 33 rue Saint Leu, 80000 Amiens, France.

E-mail address: fdurand@u-picardie.fr

Departamento de Ingeniería Matemática, Universidad de Chile and Centro de Modelamiento Matemático, UMR 2071 UChile-CNRS, Casilla 170/3 Correo 3, Santiago, CHILE.

E-mail address: amaass@dim.uchile.cl 